\title{
User Assistance for Effective Data Mining
}

\author{
Pankaj S. Kulkarni \\ Lecturer, Computer Engg. \\ Dept., Shatabdi Institute of \\ Technology, Nashik,India.
}

\author{
Varsha C. Belokar \\ Lecturer, Computer Engg. \\ Dept., Shatabdi Institute of \\ Technology, \\ Nashik, India.
}

\author{
S. S. Sane, $\mathrm{PhD}$ \\ H.O.D. Comp. Engg. Dept., K. \\ K. Wagh Institute of \\ Engineering, Education \& \\ Research, Nashik, \\ Maharashtra, India
}

\begin{abstract}
Today, several tools are available for solving data mining problems, both in open source and commercial category. For solving classification problems these tools provide variety of strategies such as decision tree, neural networks, lazy classifiers etc. For each strategy, the tools allow the user to select specific values for large number parameters ${ }^{[1]}$ for e.g. in case of a neural network classifier, user needs to provide values for parameters such as epochs, learning rate, momentum etc . Although default setting for such parameters is provided by tools, it is often found that the classifier performance (accuracy) may be enhanced by making series of experiments with different values for these parameters. Thus, for a novice user it is difficult to guess proper values for these parameters and the only option is to try with series of experiments which is time consuming. This paper aims at developing a database to record the nature of data such as number and type of attributes, presence or absence of missing values etc along with various values for building classifier models and the accuracy of the classifier. Such a data is then can be made available to novice users to build a model based on past experience. The work also aims at developing required forms reports
\end{abstract}

Keywords- Data Mining, classification, classifier, filter, WEKA etc.

\section{INTRODUCTION}

In the real world we are overwhelmed with data. The amount of data in the real world, in our lives seems to go on and on increasing and there is no end insight. Most of the information is in raw form data. There is a huge amount of information that is hidden in the raw data. ${ }^{[1]}$

Data mining is the extraction of implicit, previously unknown, and potentially useful information from data. The idea is to build computer program that sift through databases automatically, seeking regularities or patterns. Several tools are available for solving data mining problems, both in open source and commercial category. ${ }^{[1][2]}$

Because of the WEKA is open source it is widely used by many organizations. ${ }^{[4]}$ WEKA provides variety of strategies such as decision tree, neural networks, lazy classifiers etc. ${ }^{[3]}$ For each strategy, the tools allow the user to select specific values for large number parameters for e.g. in case of a neural network classifier, user needs to provide values for parameters such as epochs, learning rate, momentum etc ${ }^{[4]}$. Although default setting for such parameter is provided by tools, it is often found that the classifier performance (accuracy) can be enhanced by making series of experiments with different values for these parameters. Thus, for a novice user it is difficult to guess proper values for these parameters and the only option is to try with series of experiments which is time consuming.

This project aims at developing a database that records performance of the classifiers and nature of data along with different values used for building classifier. This information is to be provided in both textual as well as graphical form so as to guide the novice users based on such past experiments.

\section{WEKA (WAIKATO ENVIRONMENT FOR KNOWLEDGE ANALYSIS) :}

WEKA was developed at the University of Waikato in New Zealand $^{[4]}[3]$. "WEKA" stands for the Waikato Environment for Knowledge Analysis ${ }^{[4]}$. The system is written in Java, an object oriented programming language that is widely available for all major computer platforms, and WEKA has been tested under Linux, Windows, and Macintosh operating systems. Java allows us to provide a uniform interface to many different learning algorithms, along with methods for pre and post processing and for evaluating the result of learning schemes on any given dataset $^{[4]}$.

WEKA provides implementations of learning algorithms that you can easily apply to your dataset. ${ }^{[4]}$ It also includes a variety of tools for transforming datasets, such as the algorithms for discretization. ${ }^{[5]}$ You can preprocess a dataset, feed it into a learning schema, and analyze the resulting classifier and its performance-all without writing any program code at all. ${ }^{[4]}$

The workbench includes methods for all the standard data mining problems: regression, classification, clustering, association rule mining, and attribute selection. All algorithms take their input in the form of a single relational table in the ARFF format, which can read from a file or generated by a database query. ${ }^{[3][4]}$

One way of using WEKA is to apply a learning method to a dataset and analyze its output to extract information about the data. Another is to apply several learners and compare their performance in order to choose one for prediction. The learning methods are called classifiers. ${ }^{[4]}$

Suppose you have some data and you want to build a decision tree from it. A common situation is for the data to be stored in a spreadsheet or database. However, WEKA expects it to be in ARFF format, because it is necessary to have type information about each attribute which cannot be automatically deduced from the attribute values. Before you can apply any algorithm to your data, it must be converted to ARFF form. ${ }^{[4]}$ This can be done very easily. Most spreadsheet and database programs allow you to export your data into a file in comma separated format-as a list of records where the items are separated by commas. Once this has been done, you need only load the file into a text editor or a word processor; add the dataset's name using the @ relation 
tag, the attribute information using @attribute, and a @ data line; save the file as raw text — and you're done! ${ }^{[4]}$

In the following example we assume that your data is stored in a Microsoft Excel spreadsheet, and you're using Microsoft Word for text processing. Of course, the process of converting data into ARFF format is very similar for other software packages. Figure 1 shows an Excel spreadsheet containing the weather data.

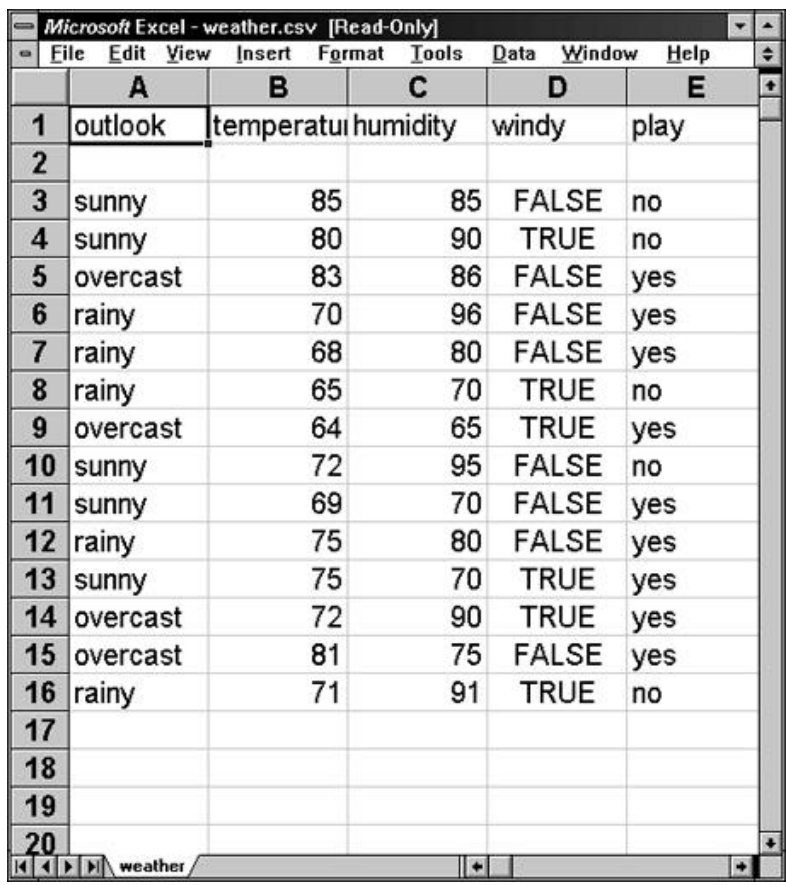

\section{Figure 1. Weather data in Microsoft Excel}

It is easy to save this data in comma-separated format. First, select the Save As... item from the File pull-down menu. Then, in the ensuing dialog box, select CSV (Comma Delimited) from the file type popup menu, enter a name for the file, and click the Save button. (A message will warn you that this will only save the active sheet: just ignore it by clicking OK.) Now load this file into Microsoft Word. Your screen will look like Figure 2. ${ }^{[3][4]}$

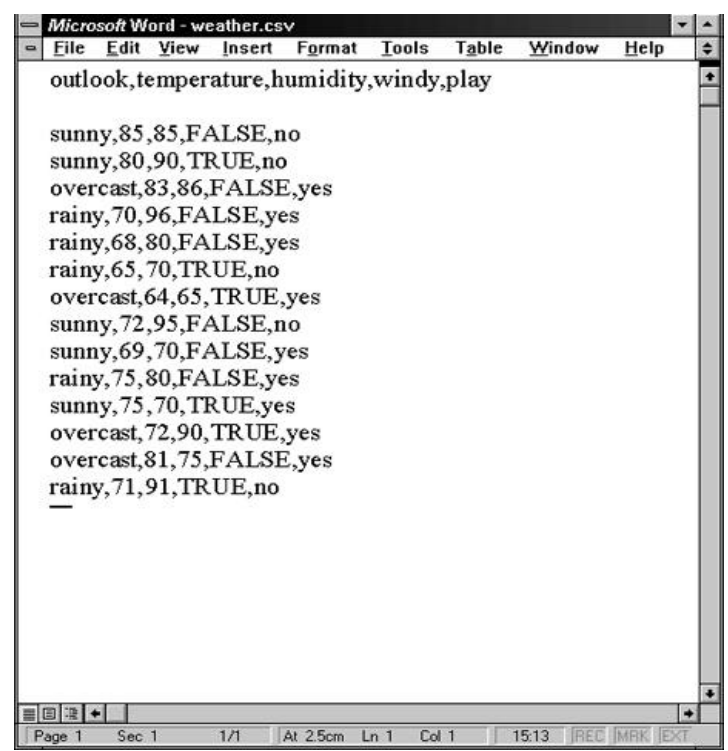

Figure 2. Weather data in CSV format
The rows of the original spreadsheet have been converted into lines of text, and the elements are separated from each other by commas. All you have to do is convert the first line, which holds the attribute names, into the header structure that makes up the beginning of an ARFF file. Figure 3 shows the result. The dataset's name is introduced by a @ relation tag, and the names, types, and values of each attribute are defined by @attribute tags. The data section of the ARFF file begins with a @ data tag. Once the structure of your dataset matches Figure3, you should save it as a text file. Choose Save as... from the File menu, and specify Text Only with Line Breaks as the file type by using the corresponding popup menu. Enter a file name, and press the Save button. Rename the file to weather.arff to indicate that it is in ARFF format. Note that the classification schemes in Weka assume by default that the class is the last attribute in the ARFF file, which fortunately it is in this case. ${ }^{[3][4]}$

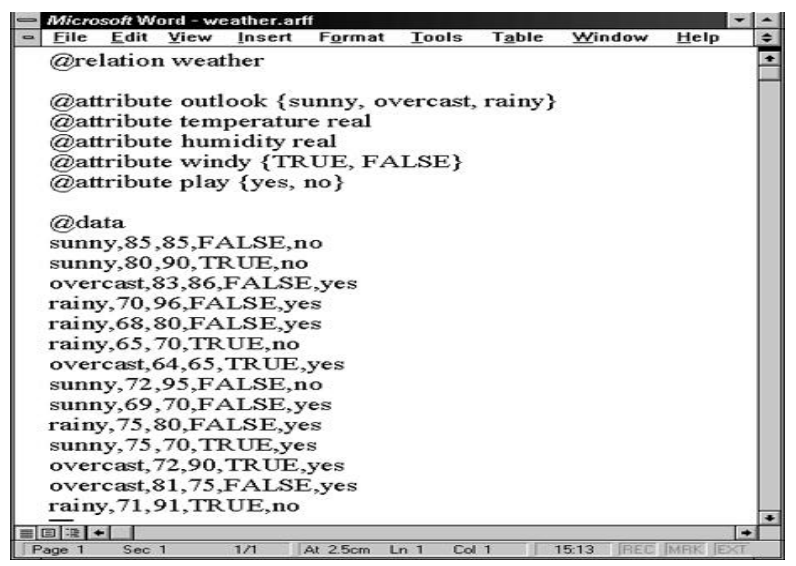

Figure 3. Weather data in ARFF format

\section{LOADING THE DATA INTO THE EXPLORER:}

Let's load this data into the Explorer and start analyzing it Fire up WEKA to get the panel shown in fig. 4 Select Explorer from the four graphical user interface choices.

\section{Weka GUI Chooser}

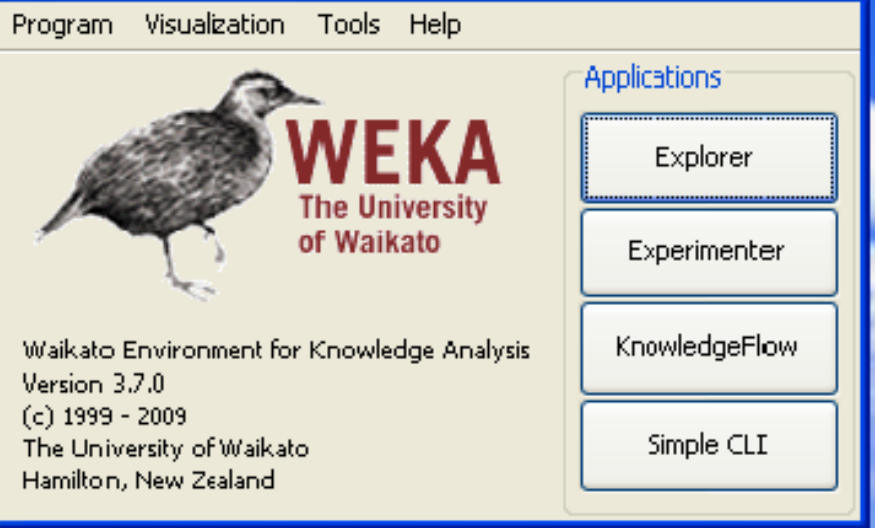

Figure 4. WEKA GUI

What you see next is the main Explorer screen, shown in fig 5. Actually, the figure shows what it will look like after you have loaded in the weather data. The six tabs along the top are basic operations that the explorer supports: 
1) Preprocess: choose the dataset and modify it in various way.

2) Classify: train learning schemes that perform classification or regression and evaluate them.

3) Cluster: learn clusters for the dataset.

4) Associate: learn association rules for the data and evaluate them.

5) Select attributes: select the most relevant aspects in the dataset.

6) Visualize: view different two-dimensional plots of the data and interact with them.

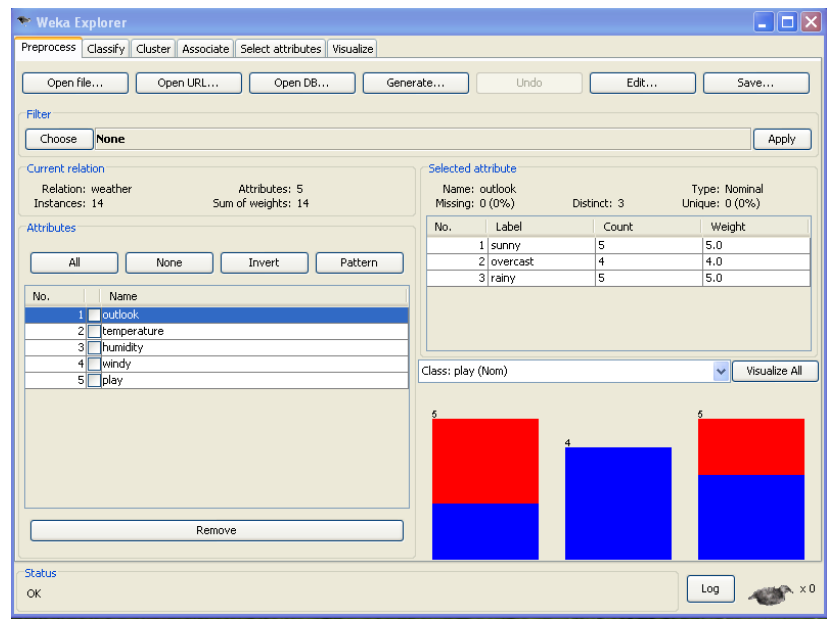

Figure 5. Weka Explorer

\section{INTERFACING WEKA WITH DATASET}

:

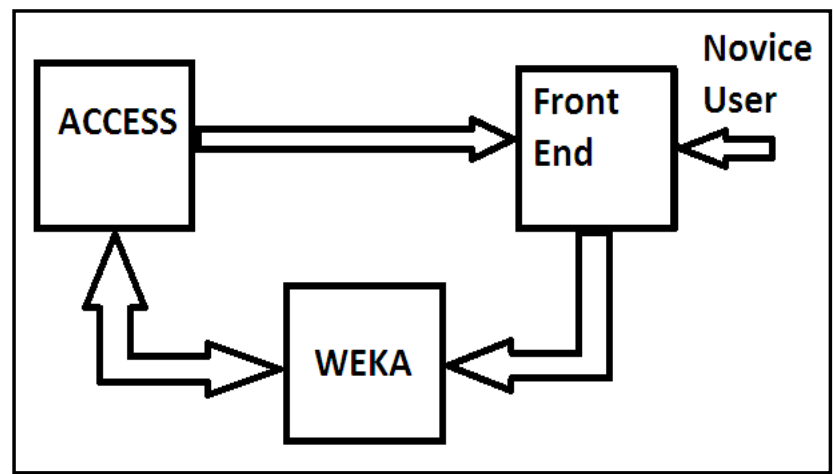

Figure 6. Applying Dataset to the WEKA

\section{UML DIAGRAMS OF PROJECT}

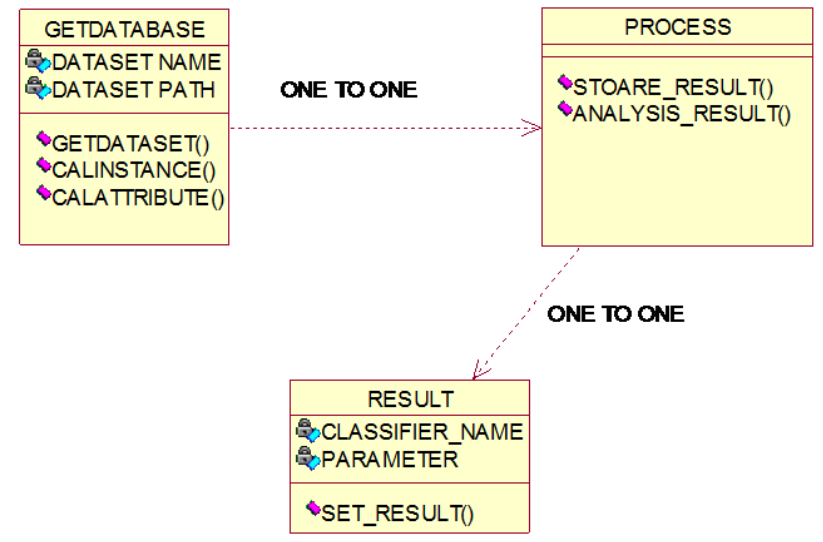

Figure 7. Class Diagram
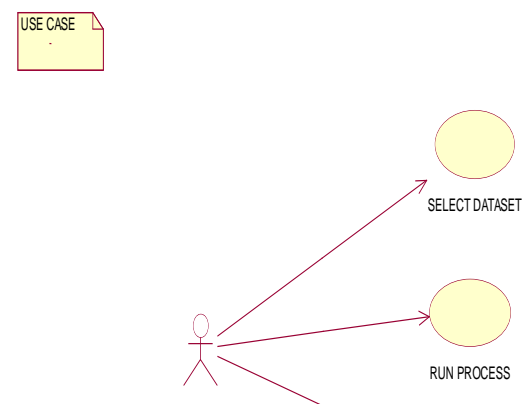

READ CLASSIFIER NAME

SEE_RESULT

READ_PARMNETER_VALUES

READ_ACCURAACY

Figure 8. Use Case Diagram

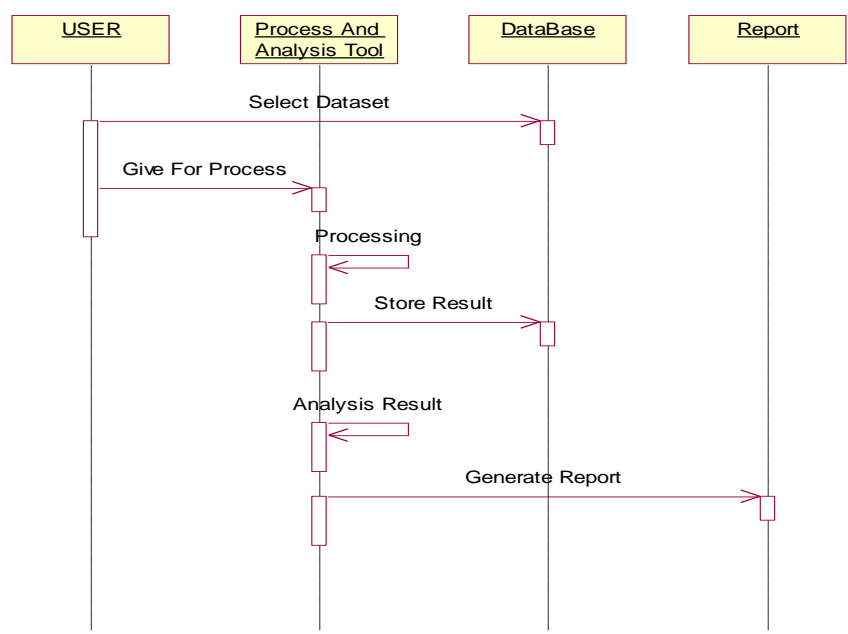

Figure 9. Sequence Diagram 


\section{DATABASE DETAILS}

This project has two database tables Dataset_Info and Classifier_Result. Dataset_Info table has 6 fields that store details about dataset. Classifier_Result table has 9 fields that store analysis result.

A. Dataset_Info:

\begin{tabular}{|c|c|c|}
\hline COLUMN NAME & CONSTRAINTS & DATA TYPE \\
\hline Id & Primary key & Number \\
\hline Dataset Name & Not Null & Varchar \\
\hline No_Attributes & Not Null & Number \\
\hline Instances & Not Null & Number \\
\hline Classes & Not Null & Number \\
\hline Missing_value_status & Not Null & Number \\
\hline
\end{tabular}

B. Classifier_Result:

\begin{tabular}{|c|c|c|}
\hline COLUMN NAME & CONSTRAINTS & DATA TYPE \\
\hline Id & Foreign Key & Number \\
\hline Classifier & Not Null & Varchar \\
\hline ParaI & Not Null & Varchar \\
\hline Para 2 & Not Null & Varchar \\
\hline$:$ & $:$ & $:$ \\
\hline ParaN & Not Null & Varchar \\
\hline Accuracy & Not Null & Number \\
\hline Time_To_Build & Not Null & Time \\
\hline
\end{tabular}

\section{RELATIONSHIP DIAGRAM}

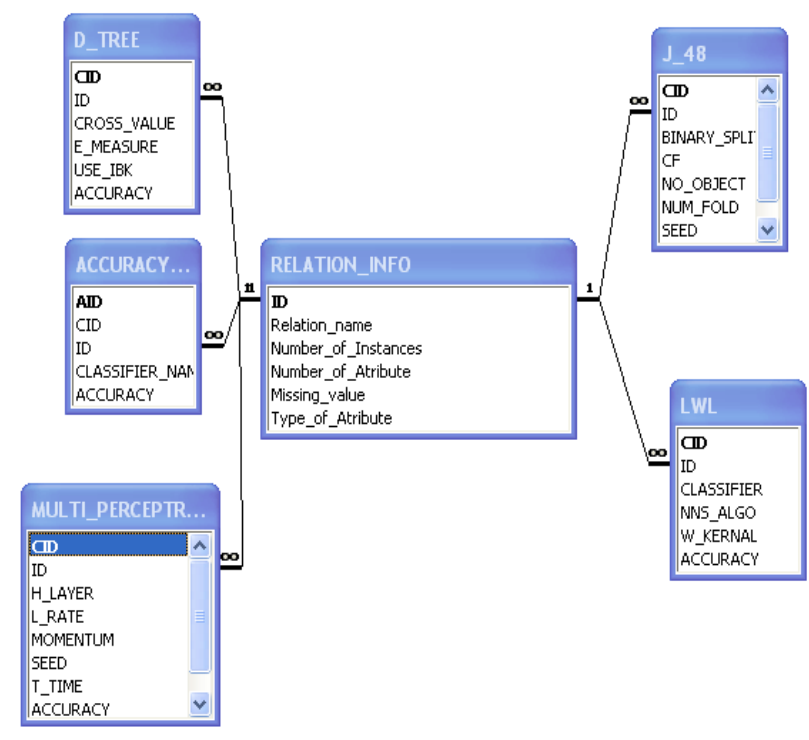

Figure $10 . \quad$ Relationship diagram

\section{CONCLUSIONS}

This project aims at developing a database to record the nature of data such as number and type of attributes, presence or absence of missing values etc along with various values for building classifier models and the accuracy of the classifier. Such a database is then can be made available to novice users to build a model based on past experience. The work also aims at developing various reports.

\section{ACKNOWLEDGEMENT}

We have great pleasure to express our deep sense of gratitude towards our parents \& Prof. Dr. Sane S. S. for their support and information. Their valuable suggestions and encouragement were the driving force for us to work with high spirit and enthusiasm and allowing us to present this paper and giving us moral support.

We would also thank Prof. Mrs. P. R. Mogal, Prof. S. R. Upasani, Prof. G. B. Katkade, Prof. N. L. Bhale, Jitendra Muradnar, Prof. H. P. Bhabad, Prof. C. R. Ghuge for their support \& encouragement. We would also thank the entire staff of computer engineering department for their support. We would also like to thank our friends and dear ones for supporting us during some tough and frustrating time and encouraging us all the way through this project.

\section{AUTHORS PROFILE}

$$
\text { Mr. Pankaj S. Kulkarni }
$$

Has completed Bachelors degree in Computer Engineering from K. K. Wagh Institute of Engineering, Education \& Research, Nashik. Pursuing M.Tech in Information Technology from Rajasthan Technical University, Kota. Currently Working as lecturer in Shatabdi Institute of Technology, Agaskhind, Nashik. Has presented more than 5 research papers at international level.

\section{Ms. V. C. Belokar}

Completed her Bachelor's degree in Information Technology from Mumbai Education Trust's Bhujbal College of Engineering, Nashik. Pursuing M.Tech in Information Technology from Rajasthan Technical University, Kota. She is presently working as lecturer in Shatabdi Institute of Technology, Agaskhind, Nashik. She has more than 5 international papers on her name.

\section{Dr. S. S. Sane}

Prof. Dr. Shirish S Sane obtained his Diploma in Electronics and Radio Engineering in the year 1984 from the Cusrow Wadia Institute of Technology, Pune and obtained his bachelor's degree in Computer Engineering from the Pune Institute of Computer Technology (PICT), Pune in the year 1987. He then worked as a Software Engineer at the "Algorhythms Computer Software Consultants", Pune. With his passion towards the field of Education, he joined the $\mathrm{K} \mathrm{K}$ Wagh Institute of Engineering Education \& Research, formerly called the K K Wagh College of Engineering, Nashik in the year 1988. He obtained his Masters Degree M. Tech in Computer Science \& Engineering from Indian Institute of Technology (IIT), Mumbai in the year 1995. Obtained $\mathrm{PhD}$ in Data Mining from Pune University. Since the year 1998, he is working as the Head of the Computer Engineering Department and the Professor Incharge of the "Kusumagraj" Central Library at the $\mathrm{K} \mathrm{K}$ Wagh Institute of Engineering Education \& Research. He has also worked as the Head of the Information Technology Department at that institute. 


\section{REFERENCE}

[1] Data Mining: A Knowledge Discovery Approach, K. Cios, W. Pedrycz, R. Swiniarski, L. Kurgan, Springer, ISBN: 978-0-387-33333-5, 2007

[2] Data Mining: Concepts, Models, Methods, and Algorithms, Mehmed Kantardzic, ISBN: 0471228524, Wiley-IEEE Press, 2002.
[3] Ian Witten and Eibe Frank, Data Mining: Practical Machine Learning Tools and Techniques, 2nd Edition, Morgan Kaufmann, ISBN 0120884070,2005.

[4] WEKA manual.

[5] Zdravko Markov, Ingrid Russell, An Introduction to the Weka DataMining System 